\title{
ZNF4 I8 overexpression protects against gastric carcinoma and prompts a good prognosis
}

This article was published in the following Dove Press journal: OncoTargets and Therapy

\author{
Hong-xia Hui ${ }^{1, *}$ \\ Zhong-wu $\mathrm{Hu}^{2, *}$ \\ Chao Jiang' \\ Jian $\mathrm{Wu}^{3}$ \\ Yong Gao' \\ Xiao-wei Wang'
}

'Department of Medical Oncology, Huai'an First People's Hospital,

Nanjing Medical University, Huai'an, Jiangsu, People's Republic of China; ${ }^{2}$ Department of Thoracic Surgery, Huai'an First People's Hospital, Nanjing Medical University, Huai'an, Jiangsu, People's Republic of China; ${ }^{3}$ Department of Pathology, Huai'an First People's Hospital, Nanjing Medical University, Huai'an, Jiangsu, People's Republic of China

*These authors contributed equally to this work
Correspondence: Xiao-wei Wang; Yong Gao

Department of Medical Oncology, Huai'an First People's Hospital,

Nanjing Medical University, 6 Beijing

Road West, Huai'an, Jiangsu 223300,

People's Republic of China

Tel +86 I39 I2073286

Email wxw7352@sina.com;

hayy_gy@163.com
Background: This study aimed to investigate the prognostic power of zinc-finger protein 418 (ZNF418) in gastric cancer (GC) and its potential role in GC development and progression.

Patients and methods: A total of $10 \mathrm{GC}$ patients' individual plasmas were collected and screened for dysregulated mRNA using human microarray. Among these dysregulated mRNAs, ZNF418 was found to be significantly downregulated in IIIA-IV stage GC patients compared to IA-IIA stage GC patients. Subsequently, the ZNF418 levels were detected by quantitative reverse transcription-polymerase chain reaction in both GC plasmas and tissues in a larger sample, and the association between ZNF418 expression level and clinicopathological features as well as overall survival (OS) of GC patients was further analyzed. Finally, a network of ZNF418 interactions with other molecules was predicated in STRING and GEPIA databases.

Results: Human mRNA microarray was performed to screen for abnormally expressed mRNAs between five IIIA-IV stage GC patients' plasma and five IA-IIA stage GC patients' plasma. A total of 662 mRNAs were differentially expressed in the IIIA-IV stage GC plasma vs IA-IIA stage GC plasma among all the candidate mRNAs according to the Student's $t$-test. Results showed that a decrease in the ZNF418 expression level was associated with the presence of GC and also with higher tumor-node-metastasis stage and lower OS rates compared with that in adjacent noncancerous tissues. Cox regression analysis results demonstrated that the OS was independently correlated with ZNF418 expression. Finally, the prediction results showed that a total of eight mRNAs might have an interaction with ZNF418 in both STRING and GEPIA databases.

Conclusion: ZNF418 was first identified to be significantly downregulated in GC. Our study indicated that ZNF418 might serve as a novel biomarker for GC and was involved in GC development.

Keywords: plasma, diagnosis, prognosis, biomarker

\section{Introduction}

Gastric cancer (GC) is the most common digestive system tumor and is still the primary cause of human death in developing countries, with its incidence continuously increasing in recent years. ${ }^{1} \mathrm{GC}$ can be divided into early $\mathrm{GC}$ and advanced GC based on the malignant degree and invasion depth. However, due to the lack of specific biomarkers for early diagnosis, the confirmed diagnosis patient was mainly in the advanced stage, which has a poor survival rate. ${ }^{2}$ Thus, it is necessary to identify appropriate molecular biomarkers for stage diagnosis and potential targets for GC therapy. ${ }^{3}$

The Cys2His2 (C2H2)-type zinc finger proteins (ZFPs) are a class of well-defined eukaryotic DNA-binding proteins. ${ }^{4}$ There are $\sim 700$ genes in the human genome 
encoding $\mathrm{C} 2 \mathrm{H} 2$ motifs, composed of 21 amino acids with a consensus sequence of CX2CX3FX5LX2HX3H. ${ }^{5}$ In C2H2type $\mathrm{ZFPs}$, there is a highly conserved consensus sequence TGE $(\mathrm{K} / \mathrm{R}) \mathrm{P}(\mathrm{Y} / \mathrm{F}) \mathrm{X}$, referred to as the $\mathrm{H} / \mathrm{C}$ link between adjacent zinc finger motifs. ${ }^{6}$ Each zinc finger can bind three to five base pair subsites specifically in the major groove of the DNA and constitutes the single largest class of transcription factors, which have been proved to be involved in tumor suppression, neoplastic transformation, cell differentiation, proliferation and apoptosis. ${ }^{7,8}$ A previous study has confirmed the expression of zinc-finger protein 418 (ZNF418) in various adult tissues, including the placenta, pancreas, kidney, liver, brain, muscle, lung and heart. ${ }^{6}$ However, up to date, no study has provided information regarding the expression patterns of ZNF418 and its function in GC.

The present study utilized human mRNA microarray analysis to screen mRNA expression profiles in GC plasmas and discovered a significantly downregulated mRNA of ZNF418. Based on the microarray findings, a series of validation experiments were performed to explore the role of ZNF418 in both gastric tissues and plasma. Then, the association between the expression level of ZNF418 and the clinicopathological features as well as overall survival (OS) of patients with GC was analyzed. Finally, a network of ZNF418 interactions with other molecules was predicated in STRING and GEPIA databases.

\section{Patients and methods mRNA microarray analysis}

CapitalBio Technology Human mRNA Array v4 was designed with four identical arrays per slide $(4 \times 180 \mathrm{~K}$ format $)$, with each array containing probes interrogating $\sim 34,000$ human mRNAs. Total RNA containing small RNA was extracted from plasmas by using the TRIzol reagent (Thermo Fisher Scientific, Waltham, MA, USA) according to manufacturer's protocol. Complementary DNA (cDNA) was produced by the Eberwine's linear RNA amplification method and subsequent enzymatic reaction. After completion of double-stranded cDNA (dsDNA) synthesis using DNA polymerase and RNase $H$, the dsDNA products were purified using a polymerase chain reaction (PCR) NucleoSpin Extract II Kit (MN) and eluted with $30 \mu \mathrm{L}$ elution buffer.

\section{Ethics statement}

The study was approved by the ethics committee of Huai'an First People's Hospital, Nanjing Medical University, China. Permission for using the information in the medical records of the patients for research purposes was obtained from the Huai'an First People's Hospital.

\section{Patients and samples}

A total of 84 pairs of GC tissues and corresponding samples were obtained from patients with GC who underwent surgery during 2008-2016 at the Huai'an First People's Hospital, Nanjing Medical University. Before the operation, the patients have not received radiotherapy or chemotherapy. These tissue specimens were immediately stored in RNAfixer reagent after removal from patients' stomachs and were kept at $-80^{\circ} \mathrm{C}$ in a refrigerator until use. Following the principle, the paired adjacent non-tumor tissues were confirmed to have no tumor cells through pathological analysis and were located $5 \mathrm{~cm}$ away from the edge of the GC. According to the tumor-node-metastasis (TNM) (V.8.2016) staging system of the International Union against Cancer, all tumors were staged accurately.

Peripheral blood ( $3 \mathrm{~mL}$ ) of $50 \mathrm{GC}$ patients was obtained before the operation, and then, the plasma was isolated. Following the age- and gender-matched criteria, fresh normal plasma samples were collected from 10 healthy people at the Huai' an First People's Hospital, Nanjing Medical University, in February 2016. All peripheral blood samples used ethylenediaminetetraacetic acid (EDTA) as the anticoagulant. Written informed consent was obtained from each patient before recruitment.

\section{Follow-up}

For the OS rate study, each patient saw his or her doctor during the follow-up period for assessment every 3 months in the first year, every 6 months in the second year and annually thereafter until December 2017. Dates of death were obtained from inpatient and outpatient records or from the patients' families through telephone follow-up. The OS was defined as the length of time between diagnosis and death or the last follow-up examination.

\section{RNA isolation, reverse transcription and quantitative real-time PCR}

Total RNA from paired tissues were extracted by using TRIzol reagent. All cDNAs were obtained with the Prime-Script ${ }^{\mathrm{TM}}$ RT-PCR kit following the manufacturer's instructions. The sequences of ZNF418 divergent primers were as follows: forward: 5'-TGGACAGAGGAAAGTCGTCAT-3' and reverse: 5'-AAAGTTCACAGCCACATCTTCA-3'. The primers' sequences of glyceraldehyde-3-phosphate dehydrogenase (GAPDH), as a control, were 5'-TCGACAG TCAGCCGCATCTTCTTT-3' and 5'-ACCAAATCCG TTGACTCCGACCTT-3'. These primers were synthesized by PrimerBank. We used quantitative reverse transcriptionpolymerase chain reaction (qRT-PCR) on the ABI7500 
System (Thermo Fisher Scientific) to measure the expression of mRNA. The PCR conditions were $95^{\circ} \mathrm{C}$ for $30 \mathrm{~s}$ and then 40 cycles of $95^{\circ} \mathrm{C}$ for $5 \mathrm{~s}$ and $60^{\circ} \mathrm{C}$ for $30 \mathrm{~s}$. Finally, annealing and extension at $95^{\circ} \mathrm{C}$ for $15 \mathrm{~s}, 60^{\circ} \mathrm{C}$ for $60 \mathrm{~s}$ and $95^{\circ} \mathrm{C}$ for $15 \mathrm{~s}$ were carried out. Determining the reproducibility of the qRT-PCR method for detecting ZNF418 levels in GC tissue, we performed qRT-PCR three times to measure differences between experimental batches.

\section{Statistical analysis}

Comparison of continuous data was analyzed using an independent Student's $t$-test between the two groups. A receiver operating characteristic (ROC) curve was established to evaluate their diagnostic values. The correction between ZNF418 expression and OS time was evaluated by KaplanMeier plots and log-rank tests. All statistical analyses were performed using SPSS for Windows v.17.0 (SPSS Inc., Chicago, IL, USA). For all results, $P<0.05$ was considered to be statistically significant.

\section{Results}

\section{mRNA expression profiles in GC}

High-throughput human mRNA microarray was conducted to assess the differences in mRNA expression profiles between five advanced GC patients' plasma and five early GC patients' plasma. A total of $662 \mathrm{mRNAs}$ were differentially expressed in IIIA-IV stage GC plasma vs IA-IIA stage GC plasma among all the candidate mRNAs according to the Student's $t$-test. Of 662 mRNAs, 69 were significantly upregulated in IIIA-IV stage GC plasma, whereas 593 were significantly downregulated. We selected a total of 10 upregulated and downregulated mRNAs from high rank to low rank based on the multiple fold difference between the expression of IIIA-IV stage GC plasma and IA-IIA stage GC plasma. Subsequently, we used qRT-PCR to detect the expression levels of these dysregulated mRNAs in GC plasma and found that compared to other mRNAs, the expression of ZNF418 was significantly increased in IIIA-IV stage GC plasma vs IA-IIA stage GC plasma, which was selected as a candidate target for GC for further study.

\section{ZNF4 I 8 was significantly downregulated in GC plasma}

The expression of ZNF418 in 40 GC plasmas was measured, and fresh normal plasma samples from 10 healthy individuals were used as control. The results indicated that ZNF418 expression was downregulated in GC plasma compared with normal cases (Figure 1A). To evaluate the potential diagnostic value of ZNF418, the ROC curve has been used, and the larger the area under the ROC curve (AUC), the higher the diagnostic value. As shown in Figure 1B, we found that in plasma, the AUC was 0.7025 (Figure 1B). Down-expression of the ZNF418 level was negatively associated with the TNM stage. Figure $1 \mathrm{C}$ describes the relationship between $Z N F 418$ level and TNM stage, and as the TNM stage increased, the expression of ZNF418 gradually decreased (Figure 1C).

\section{ZNF4 I 8 was significantly downregulated in $\mathrm{GC}$ tissues}

To further test the expression level of ZNF418, qRT-PCR was used to measure its expression in 84 paired primary cancerous and adjacent noncancerous tissues from GC patients. The results indicated that ZNF418 expression was significantly downregulated than those in corresponding non-tumorous tissues (Figure 2A). As shown in Figure 2B, the ratio of ZNF418 low expression was $61 / 84$ (72.6\%), while the ratio of ZNF418 high expression was 23/84 (27.4\%; Figure 2B). We further searched the ZNF418 expression in the GEPIA database and found that the expression of ZNF418 in 408 GC tissues was much lower than that in 211 noncancerous tissues, consistent with our findings (Figure 2C). Figure 2D reveals that the AUC was 0.7060 .

As shown in Table 1, abnormal expression of ZNF418 level was not aberrantly associated with age, gender, diameter, differentiation or lymph node metastasis in patients with GC (Table 1). However, down-expression of ZNF418 level was negatively associated with $\mathrm{T}$ stage and TNM stage, and Figure 2E describes the relationship between ZNF418 level and TNM stage in more detail.

\section{ZNF4 I 8 was correlated with OS of patients with GC}

Kaplan-Meier survival curves indicated that patients with low ZNF418 expression exhibited a greater probability of attaining shorter OS (Figure 3) than those with high ZNF418 expression. The mean OS time in patients with low expression for ZNF418 was 38 months, which was significantly lower than that in patients with high expression for ZNF418 (57 months). Cox regression analysis showed that survival was independently correlated with ZNF418 expression $(P=0.043 ;$ Table 2$)$.

\section{Prediction and annotation of ZNF4 I 8 with other molecules' network}

To identify the other molecules that might have an association with ZNF418, we performed an interaction network prediction on the STRING database (https://string-db.org) and further forecast in the GEPIA database (http://gepia.cancer-pku.cn/index.html) 


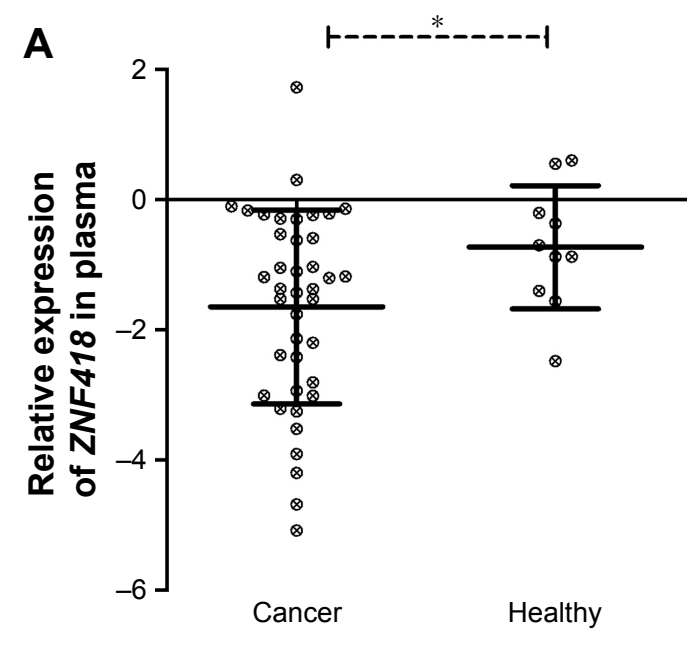

B
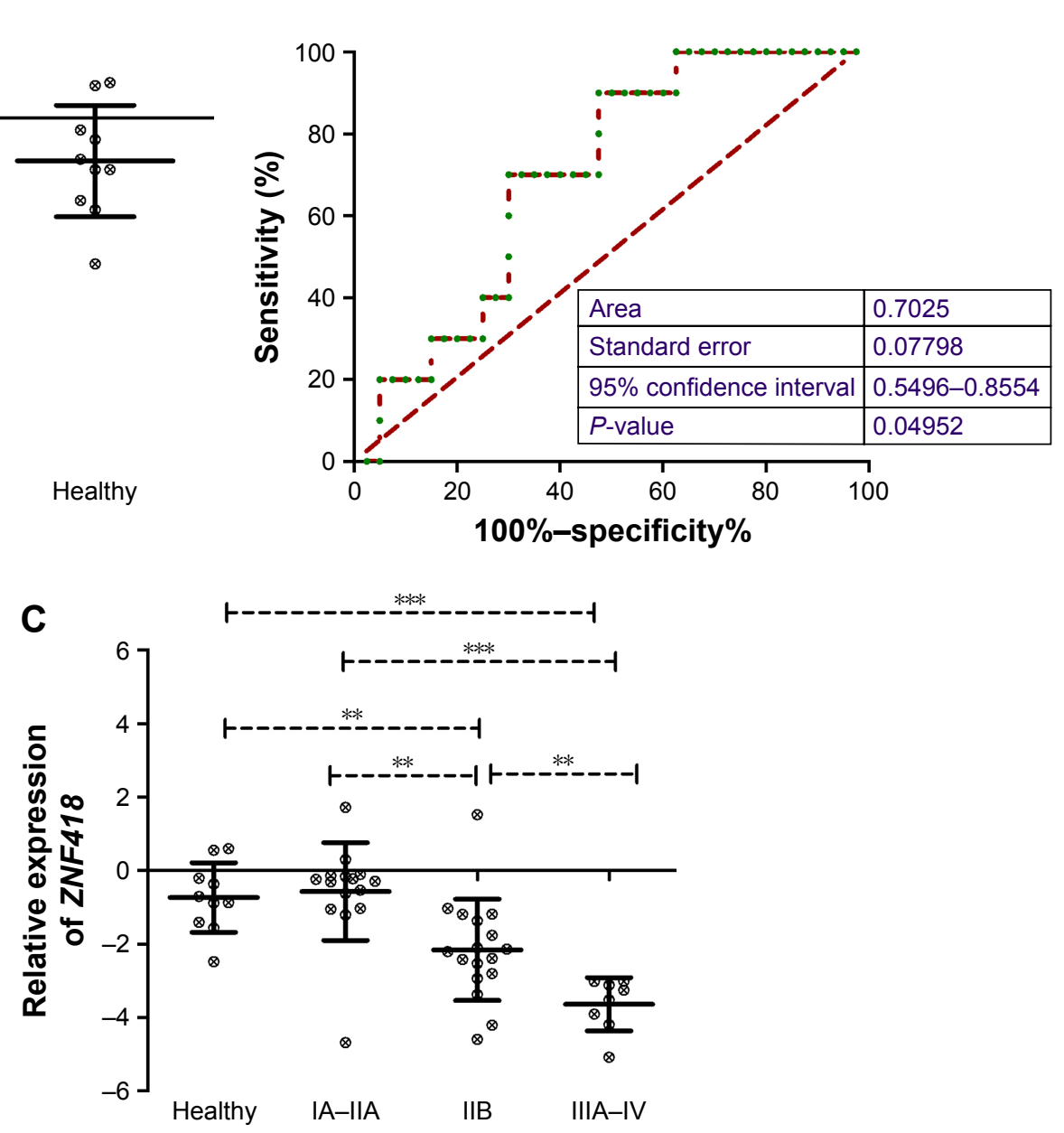

Figure I ZNF4 I 8 was significantly downregulated in GC plasma compared to healthy cases.

Notes: (A) ZNF4I8 expression was downregulated in GC plasma compared with normal cases. (B) The AUC was 0.7025 . (C) Down-expression of ZNF4I8 level was negatively associated with TNM. $* P<0.05$, $* * P<0.01$ and $* * * P<0.001$.

Abbreviations: ZNF4/8, zinc-finger protein 4I8; GC, gastric cancer; AUC, area under the ROC curve; TNM, tumor-node-metastasis; ROC, receiver operating characteristic.

to increase the credibility of the conclusion. A total of 10 candidate mRNAs (KIAA1984 with another name of CCDC183, PPP1R37, POTEE, POTEI, POTEJ, POTEF, TRIM28, AGAP4, RIPK4, PHLPP2) might have an interaction with
ZNF418 in this study (Figure 4A). Subsequently, we look up the relationship between $Z N F 418$ and 10 molecules in the GEPIA database. We used the non-log scale for calculation and the log-scale axis for visualization. The results revealed
A

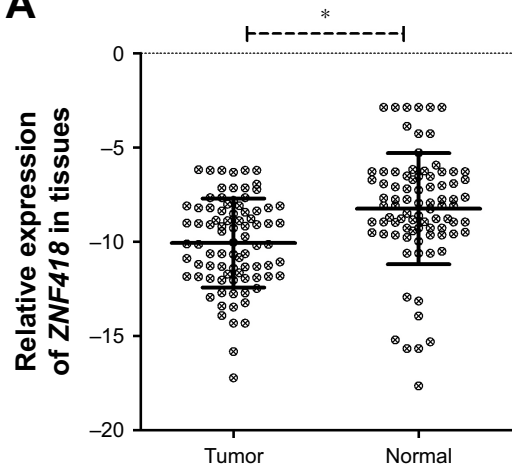

B

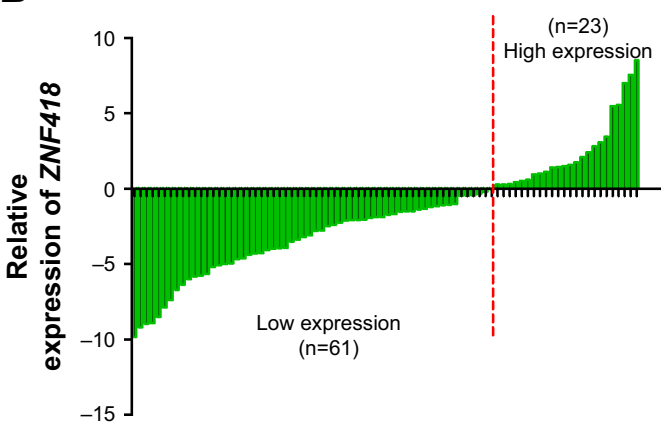

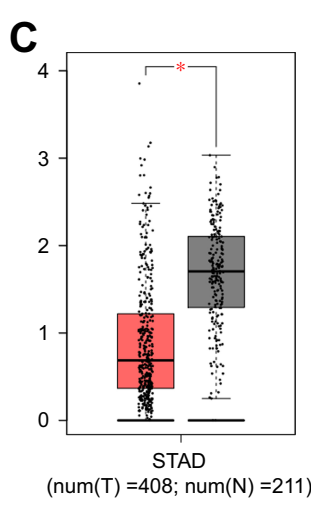

Figure 2 (Continued) 
D

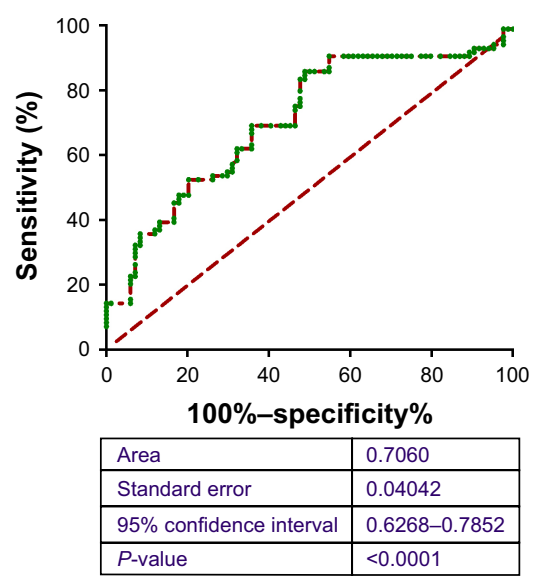

E

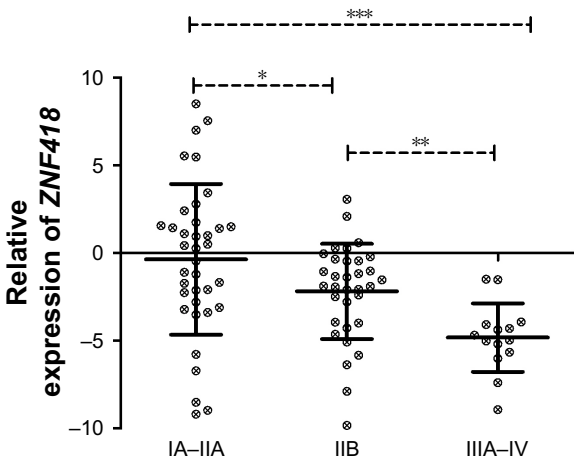

Figure 2 ZNF4 I 8 was significantly downregulated in GC tissues compared to adjacent noncancerous tissues.

Notes: (A) ZNF4 I 8 expression was downregulated in GC tissues. (B) The ratio of ZNF4 I8 low expression was $61 / 84$ (72.6\%), while the ratio of ZNF4 I 8 high expression was $23 / 84$ (27.4\%). (C) The GEPIA database revealed that ZNF4 I 8 expression was significantly downregulated in GC tissues. (D) The AUC was 0.7060 . (E) Down-expression of ZNF4 I 8 level was negatively associated with TNM. *P<0.05, **P<0.0I and ***P<0.00I.

Abbreviations: ZNF4/8, zinc-finger protein 4I8; GC, gastric cancer; TNM, tumor-node-metastasis; AUC, area under the ROC curve; ROC, receiver operating characteristic; STAD, stomach adenocarcinoma.

that the forecasts for both database sites were consistent except for PPP1R37 and POTEJ (Figure 4B).

\section{Discussion}

Early detection and disease progression monitoring remain the effective treatment against GC. Novel GC detection and

Table I The relationship between ZNF4I8 expression levels $\left(-\Delta \Delta C_{t}\right)$ in $\mathrm{GC}$ tissues and clinicopathological factors of patients

\begin{tabular}{|c|c|c|c|}
\hline Variables & No of patients & Mean \pm SD & $P$-value \\
\hline \multicolumn{3}{|l|}{ Age (years) } & 0.133 \\
\hline$\geq 60$ & 70 & $-2.09 \pm 3.64$ & \\
\hline$<60$ & 14 & $-0.44 \pm 4.09$ & \\
\hline \multicolumn{3}{|l|}{ Gender } & $0.26 \mathrm{I}$ \\
\hline Female & 38 & $-2.33 \pm 3.73$ & \\
\hline Male & 46 & $-1.40 \pm 3.74$ & \\
\hline \multicolumn{3}{|l|}{ Diameter $(\mathrm{cm})$} & 0.237 \\
\hline$\geq 5$ & 57 & $-2.15 \pm 3.53$ & \\
\hline$<5$ & 27 & $-1.11 \pm 4.13$ & \\
\hline \multicolumn{3}{|l|}{ Differentiation } & 0.293 \\
\hline Low-middle & 69 & $-2.02 \pm 3.56$ & \\
\hline High & 15 & $-0.89 \pm 4.52$ & \\
\hline \multicolumn{3}{|l|}{ T stage } & $0.000 *$ \\
\hline TI-T2 & 37 & $-0.35 \pm 4.30$ & \\
\hline $\mathrm{T} 3$ & 33 & $-2.18 \pm 2.72$ & \\
\hline $\mathrm{T} 4 \mathrm{a}-\mathrm{T} 4 \mathrm{~b}$ & 14 & $-4.82 \pm 1.95$ & \\
\hline \multicolumn{3}{|c|}{ Lymphatic metastasis } & 0.285 \\
\hline No & 14 & $-2.80 \pm 4.42$ & \\
\hline Yes & 70 & $-1.62 \pm 3.60$ & \\
\hline \multicolumn{3}{|l|}{ TNM stage } & $0.000 *$ \\
\hline IA-IIA & 37 & $-0.35 \pm 4.30$ & \\
\hline IIB & 33 & $-2.18 \pm 2.72$ & \\
\hline IIIA-IV & 14 & $-4.82 \pm 1.95$ & \\
\hline
\end{tabular}

Note: $* P<0.05$

Abbreviations: ZNF4 I8, zinc-finger protein 4|8; GC, gastric cancer; TNM, tumornode-metastasis. monitoring methods are urgently needed because of current biomarkers, such as CA199 or CEA, lacking enough specificity. Zinc finger-containing transcription factors have been implicated as critical regulators of multiple cardiacexpressed genes and are thought to be important for various human diseases including GC and development. A previous study reported that ZNF143 was overexpressed in specimens of GC compared with adjacent normal tissues and ectopic overexpression of ZNF143 enhanced GC migration, whereas ZNF143 knockdown suppressed this effect in vitro. Moreover, the authors of that study found that ZNF143 enhanced GC cell migration by promoting the process of epithelialmesenchymal transition (EMT) through the PI3K/AKT signaling pathway. ${ }^{9}$ In addition to this, Chen et a ${ }^{10}$ found

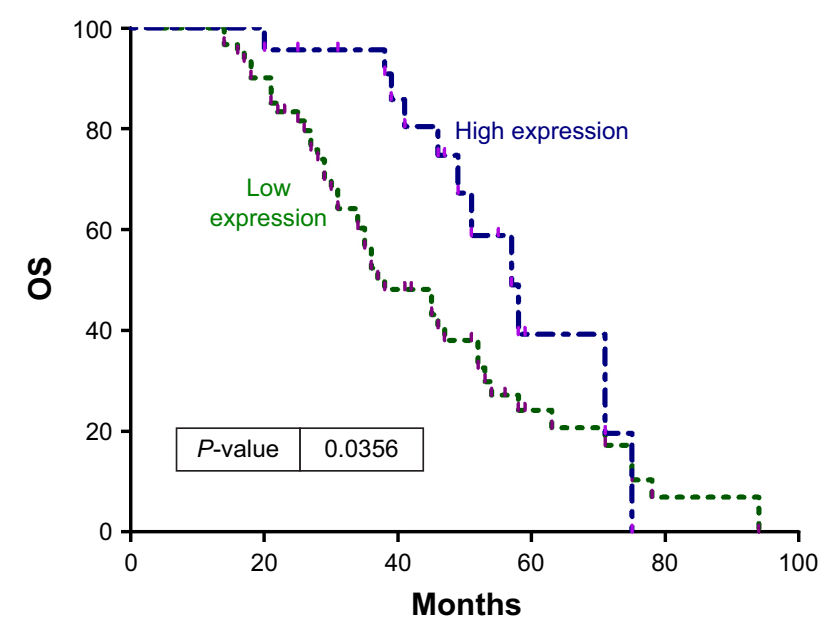

Figure 3 ZNF4 8 was correlated with OS of patients with GC.

Abbreviations: ZNF4/8, zinc-finger protein 4I8; OS, overall survival; GC, gastric cancer. 
Table 2 Cox regression analysis of factors associated with OS in GC

\begin{tabular}{lllll}
\hline Factor & $\begin{array}{l}\text { Hazard } \\
\text { ratio }\end{array}$ & P-value & $\begin{array}{l}\text { 95\% confidence } \\
\text { interval }\end{array}$ \\
\cline { 3 - 5 } & & & $\begin{array}{l}\text { Lower } \\
\text { limitation }\end{array}$ & $\begin{array}{l}\text { Upper } \\
\text { limitation }\end{array}$ \\
\hline Gender & 0.729 & 0.259 & 0.422 & 1.261 \\
Age & 1.511 & 0.228 & 0.773 & 2.954 \\
Diameter & 1.205 & 0.522 & 0.681 & 2.132 \\
Differentiation & 1.094 & 0.809 & 0.527 & 2.270 \\
T stage & 1.270 & 0.184 & 0.893 & 1.805 \\
Lymphatic metastasis & 1.837 & 0.054 & 0.321 & 1.224 \\
TNM stage & 2.213 & $0.000^{*}$ & 1.487 & 3.293 \\
ZNF4 I8 expression & 0.500 & $0.043^{*}$ & 0.255 & 0.978 \\
\hline
\end{tabular}

Note: $* p<0.05$.

Abbreviations: OS, overall survival; GC, gastric cancer; TNM, tumor-nodemetastasis; ZNF4 I8, zinc-finger protein 4I8.

that knockdown of zinc-finger E-box-binding homeobox 1 (ZEB1) expression by RNA interference can decrease lncRNA HOTAIR expression and restrain cell proliferation, invasion and migration in GC cells.

With regard to $Z N F 418$, no relevant research on GC was found. However, $\mathrm{Li}$ et $\mathrm{al}^{8}$ reported that overexpression of ZNF418 in COS-7 cells inhibited the transcriptional activity of SRE and AP-1, which may be silenced by siRNA and may act as a negative regulator in MAPK signaling pathway. Pan et $\mathrm{al}^{11}$ found that ZNF418 was markedly downregulated in hearts of cardiac hypertrophy and hypertrophic primary cardiomyocytes and downregulated ZNF418 exacerbated the myocyte size and fibrosis and moreover increased the mRNA levels of ANP, BNP, $\beta$-MHC, MCIP1.4, collagen 1a, collagen III, MMP-2 and fibronectin in hearts of AB-treated ZNF418 knockout mice or Ang II-treated cardiomyocytes with ZNF418.

In our study, microarray revealed that ZNF418 was involved in the progression and development of GC. The results showed that ZNF418 expression was downregulated in GC plasma compared with normal cases with an AUC of 0.7025 , which was consistent with the study of Pan et al. ${ }^{11}$ Down-expression of ZNF418 level was negatively associated with the TNM stage, which exactly matched the result of the microarray. Besides, qRT-PCR was used to measure its expression in 84 paired primary cancerous and adjacent noncancerous tissues from GC patients. The results showed that ZNF418 expression was significantly downregulated than that in corresponding non-tumorous tissues. To further
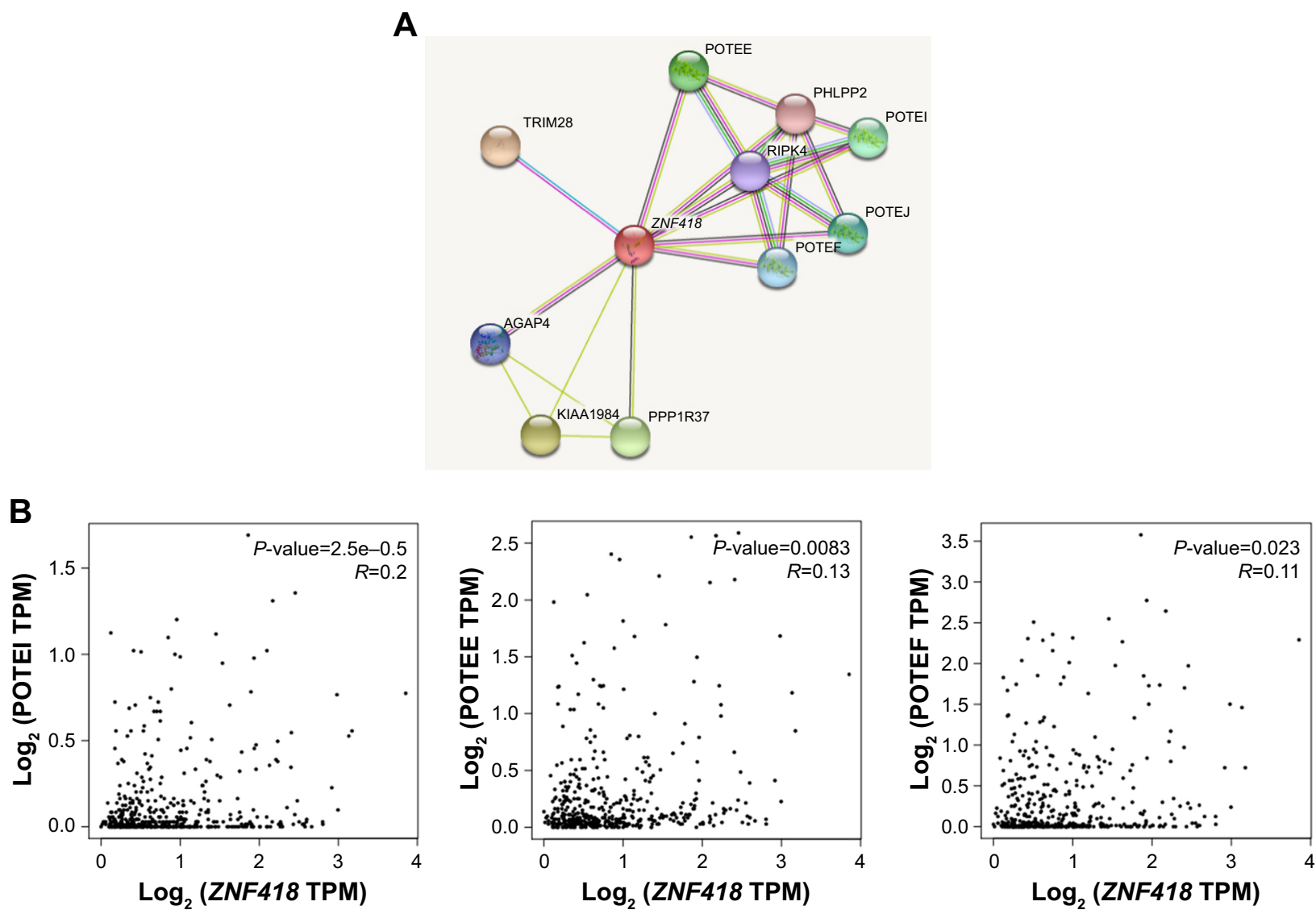

Figure 4 (Continued) 

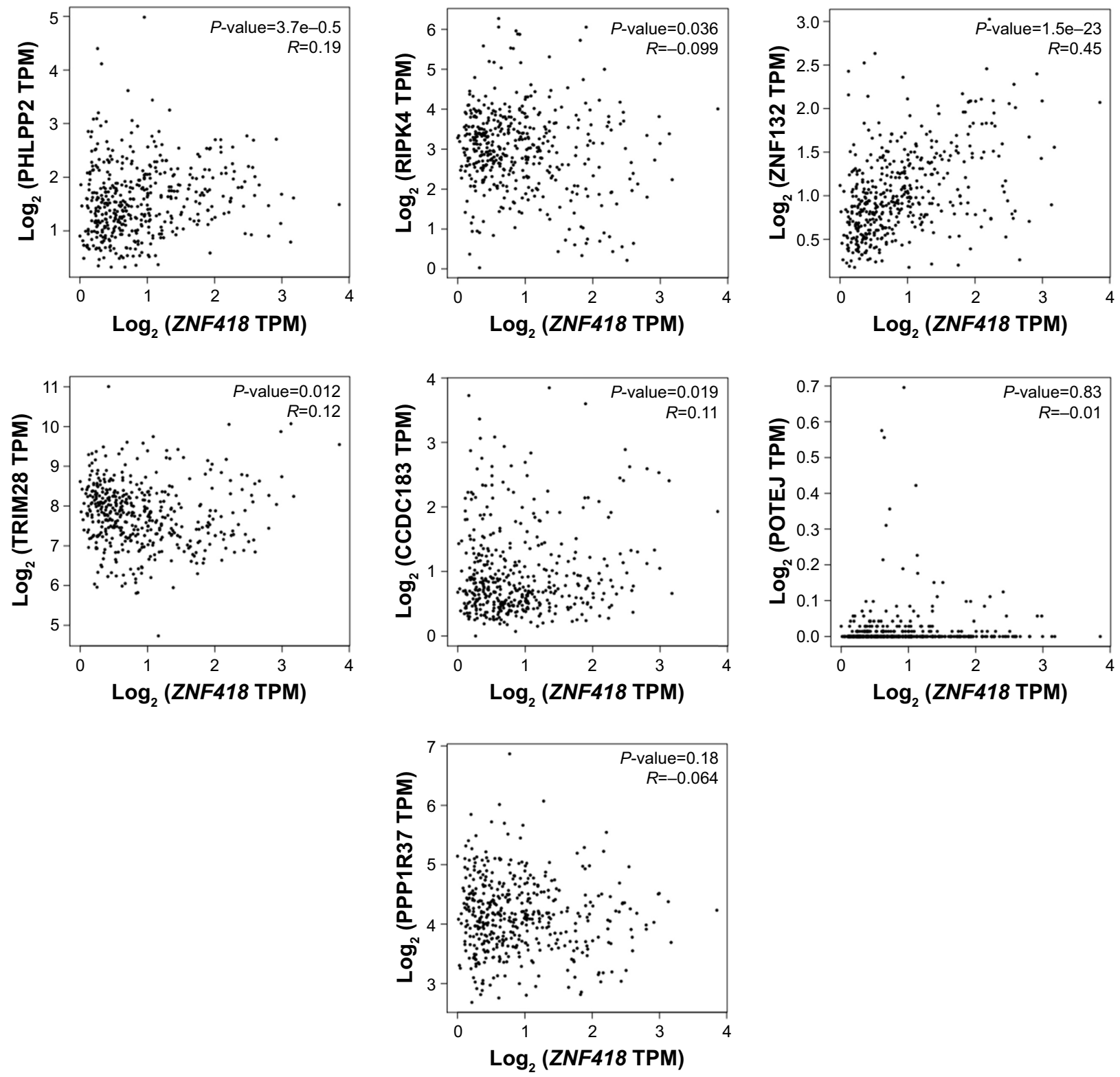

Figure 4 Network prediction and annotation of ZNF4/8 with other molecules.

Notes: (A) Ten candidate mRNAs might have an interaction with ZNF4 I8. (B) The GEPIA database revealed that PPPIR37 or POTEJ might not be associated with ZNF4I8. Abbreviations: ZNF4/8, zinc-finger protein 4I8; TPM, transcripts per million.

confirm our results, we searched the ZNF418 expression in the GEPIA database and found that the expression of ZNF418 in 408 GC tissues was much lower than that in 211 noncancerous tissues, which was consistent with our findings. Moreover, clinical analysis indicated that ZNF418 expression was significantly downregulated than that in corresponding non-tumorous tissues and down-expression of ZNF418 level was negatively associated with the TNM stage as well. Kaplan-Meier survival curves indicated that patients with low ZNF418 expression exhibited a greater probability of attaining shorter OS than those with high ZNF418 expression. This result suggested the possible association of ZNF418 down-expression with poor clinical prognosis. In addition to ZNF418 expression, tumor stage was also a significant predictor of OS. These results were supported further in Cox regression analysis, suggesting that the ZNF418 may be involved in the invasion and progression of human GC. The current studies have provided evidences that ZNF418 might serve as a novel biomarker for GC and was involved in $\mathrm{GC}$ development.

To identify the other molecules that might have an association with ZNF418, we performed network prediction and annotation of ZNF418 with other molecules in the STRING database and further forecast in the GEPIA database. A total 
of 10 candidate mRNAs showed a close interaction with ZNF418 in the STRING database. However, when we subsequently looked them up in the GEPIA database one by one, we found that only eight mRNAs might be associated with ZNF418 except for PPP1R37 and POTEJ. Overall, the predictive value in the database plays an important role in our understanding of the linkages between ZNF418 and other molecules. Although the present study shows inconsistencies in the predictions of the two databases, most of the results are still consistent and valid. Multi-database predictions and joint analyses enhance the credibility of the conclusions and provide clues and directions for our follow-up studies.

Several limitations should not be ignored when interpreting the results. First, all blood samples did not correspond to tissue samples and if the follow-up study can be the two together, the research will be of greater significance. Second, all cases were from one hospital and our results were limited to one location. Therefore, more studies containing more locations of possible differences are needed for further study. Third, this study did not do more in-depth functional studies but drew conclusions only from data analysis. As a result, further biologic and functional evidence is needed to confirm the function of ZNF418 in GC.

\section{Conclusion}

We first identified that ZNF418 was significantly downregulated in GC. Our study indicated that ZNF418 might serve as a novel biomarker for GC and was involved in gastric carcinoma development. However, the detailed molecular mechanism by which ZNF418 contributed to GC proliferation, invasion and metastasis requires further research.

\section{Disclosure}

The authors report no conflicts of interest in this work.

\section{References}

1. Torre LA, Bray F, Siegel RL, et al. Global cancer statistics, 2016. CA Cancer J Clin. 2016;66(1):7-30.

2. Ferlay J, Soerjomataram I, Dikshit R, et al. Cancer incidence and mortality worldwide: sources, methods and major patterns in GLOBOCAN 2012. Int J Cancer. 2015;136(5):E359-E386.

3. Pan L, He M, Ma J, et al. Phase and size controllable synthesis of NaYbF4 nanocrystals in oleic acid/ionic liquid two-phase system for targeted fluorescent imaging of gastric cancer. Theranostics. 2013;3(3):210-222.

4. Klug A, Schwabe JWR. Protein motifs 5. Zinc fingers. FASEB J. 1995; 9(8):597-604

5. Lander ES, Linton LM, Birren B, et al; International Human Genome Sequencing Consortium. Initial sequencing and analysis of the human genome. Nature. 2001;409(6822):860-921.

6. Dang DT, Pevsner J, Yang VW. The biology of the mammalian Kruppellike family of transcription factors. Int J Biochem Cell Biol. 2000; 32(11-12):1103-1121.

7. Nolte RT, Conlin RM, Harrison SC, Brown RS. Differing roles for zinc finger in DNA recognition: structure of a six-finger transcription factor IIIA complex. Proc Natl Acad Sci U S A. 1998;95(6):2938-2943.

8. Li Y, Yang D, Bai Y, et al. ZNF418, a novel human KRAB/C2H2 zinc finger protein, suppresses MAPK signaling pathway. Mol Cell Biochem. 2008;310(1-2):141-151.

9. Wei S, Wang L, Zhang L, et al. ZNF143 enhances metastasis of gastric cancer by promoting the process of EMT through PI3K/AKT signaling pathway. Tumour Biol. 2016;37(9):12813-12821.

10. Chen D, Chu Y, Zheng Q. Knock-down of ZEB1 inhibits the proliferation, invasion and migration of gastric cancer cells. $X i \mathrm{Bao} Y u$ Fen Zi Mian Yi Xue Za Zhi [Chin J Cell Mol Immunol]. 2017;33(8): 1073-1078.

11. Pan L, Sheng M, Huang Z, et al. Zinc-finger protein 418 overexpression protects against cardiac hypertrophy and fibrosis. PLoS One. 2017; 12(10): 0186635 .
OncoTargets and Therapy

\section{Publish your work in this journal}

OncoTargets and Therapy is an international, peer-reviewed, open access journal focusing on the pathological basis of all cancers, potential targets for therapy and treatment protocols employed to improve the management of cancer patients. The journal also focuses on the impact of management programs and new therapeutic agents and protocols on

\section{Dovepress}

patient perspectives such as quality of life, adherence and satisfaction. The manuscript management system is completely online and includes a very quick and fair peer-review system, which is all easy to use. Visit http://www.dovepress.com/testimonials.php to read real quotes from published authors. 